\title{
SREBP-1 inhibitor Betulin enhances the antitumor effect of Sorafenib on hepatocellular carcinoma via restricting cellular glycolytic activity
}

Fan Yin ${ }^{1}$, Fan Feng ${ }^{2}$, Lei Wang ${ }^{3}$, Xiaoning Wang ${ }^{4}$, Zongwei $\mathrm{Li}^{3}$ and Yu Cao $\mathbb{B}^{5}$

\begin{abstract}
Lipid metabolism that correlates tightly to the glucose metabolic regulation in malignant cells includes hepatocellular carcinoma (HCC) cells. The transcription factor Sterol Regulatory Element Binding Protein 1 (SREBP-1), a regulator of fatty acid synthesis, has been shown to pivotally regulate the proliferation and metastasis of HCC cells. However, the intrinsic mechanism by which SREBP-1 regulates the survival of HCC cells remains unclear. In this study, among HCC patients who had dismal responses to Sorafenib, a high SREBP-1 level was found in the tumors and correlated to poor survival. This observation suggested the negative role of SREBP-1 in clinical HCC prognosis. Our mechanistical studies reveal that the inhibition of SREBP-1 via its inhibitor Betulin suppresses cellular glucose metabolism. In addition to the reduced glycolytic activity, a thwarted metastatic potential was observed in HCC cells upon Betulin administration. Moreover, our data show that SREBP-1 inhibition facilitated the antitumor effects of Sorafenib on HCC cells and xenograft tumors.
\end{abstract}

\section{Introduction}

In China, the population of chronic asymptomatic Hepatitis B Virus (HBV) infection-associated patients exceeds 80 million $^{1-3}$. Hepatocellular carcinoma (HCC) is the final malignant disease developed from liver lesions such as HBV infection; it is a fatal threat to life and an increasing burden to the public medical service ${ }^{4-6}$. Thus, it is urgent to develop effective therapeutic approaches for HCC. At present, kinase inhibitors represented by Sorafenib are the major treatment choice for advanced HCC patients $^{7,8}$. As an oral administrative multi-target proteinkinase inhibitor, Sorafenib downregulates the activity of

Correspondence: Fan Yin (yinfancying@163.com) o

Yu Cao (Yu.Cao@moffitt.org)

${ }^{1}$ Department of Oncology, the Second Medical Centre \& National Clinical Research Center of Geriatric Disease, Chinese PLA General Hospital, 100853 Beijing, People's Republic of China

${ }^{2}$ Center for Clinical Laboratory, the Fifth Medical Centre, Chinese PLA General Hospital, 100039 Beijing, People's Republic of China

Full list of author information is available at the end of the article.

These authors contributed equally: Fan Yin, Fan Feng

Edited by C. Munoz-Pinedo
RTKs (receptor protein tyrosine kinases include vascular endothelial growth factor receptor $2 / 3$ [VEGFR-2/3], the hepatocyte factor receptor [c-Kit], Fms-like tyrosine kinase [FLT-3], and platelet-derived growth factor receptor- $\beta$ [PDGFR- $\beta]$ ) and inhibits the proliferation, metastasis, and angiogenesis of HCC cells ${ }^{9-11}$. The randomized controlled phase III trials, represented by the SHARP (Sorafenib HCC Assessment Randomized Protocol) clinical trial, have shown that Sorafenib significantly prolongs the median survival of patients compared with the placebo group, making it a first-line antitumor agent for advanced HCC treatment ${ }^{12-15}$. However, the sensitivity of Sorafenib in HCC patients varies, with a large proportion finally developed for Sorafenib resistance unexpectedly ${ }^{16,17}$. Thus, it is of great importance to discover a new drug and/or to develop a novel therapeutic strategy that increases the efficacy of Sorafenib.

Cellular metabolisms related to ATP production in malignant cells are more active than normal cells ${ }^{18}$. HCC cells have exaggerated glucose uptake capability for their intensive anaerobic glycolysis which provides them

\section{(c) The Author(s) 2019}

(c) (i) Open Access This article is licensed under a Creative Commons Attribution 4.0 International License, which permits use, sharing, adaptation, distribution and reproduction cc) in any medium or format, as long as you give appropriate credit to the original author(s) and the source, provide a link to the Creative Commons license, and indicate if changes were made. The images or other third party material in this article are included in the article's Creative Commons license, unless indicated otherwise in a credit line to the material. If material is not included in the article's Creative Commons license and your intended use is not permitted by statutory regulation or exceeds the permitted use, you will need to obtain permission directly from the copyright holder. To view a copy of this license, visit http://creativecommons.org/licenses/by/4.0/. 
enough energy to survive in harsh tumor microenvironment ${ }^{19,20}$. The chemoresistance of HCC cells closely correlates to the cellular metabolism; through the overwhelming anaerobic glycolytic activity, the accommodation of metabolites and the enhanced epithelial-mesenchymal transition (EMT) afterward take place $^{21,22}$. Thus, glucose metabolism is a potential target of developing antagonistic therapy for chemoresistance.

It is known that $60 \%$ of the carbons in glucose are used for fatty acid synthesis, and lipid metabolism also plays vital roles in regulating glucose uptake and glycolytic activities $^{23}$. SREBP-1 is a core transcription factor in lipid metabolism; it induces the transcription of a series of genes involved in fatty acid and triglyceride synthesis ${ }^{24}$. Inhibition of the activity of SREBP-1 decreased the synthesis and accommodation of fat and impeded the glucose uptake $\mathrm{e}^{25-27}$. Betulin belongs to lupane-type pentacyclic triterpenoids, which are widely found in plants such as Pulsatilla chinensis (Bunge) Regel; they have multiple biological functions, including antitumor activity $^{28-30}$. Betulin directly binds to the SCAP (SREBP cleavage-activating protein) to inhibit the cleavage and activation of SREBP- $1^{31,32}$. In the present study, we performed gene expression analysis on HCC patients after Sorafenib treatment, and found that the high SREBP-1 expression correlates to a poor clinical outcome. Mechanistically, our results indicate that SREBP-1 inhibition represses the cellular glycolytic activity of HCC cells, and restricts the metastasis of those malignant cells both in vitro and in vivo. We also found that Betulin works synergistically with Sorafenib on s.c. and in situ HCC tumors. Our study suggests that the SREBP-1 inhibitor and Sorafenib combination can be a novel therapeutic option for advanced HCC treatment.

\section{Materials and methods \\ Cell lines and reagents}

The hepatic cell lines: non-tumor cell line L-02, HCC cell lines HepG2, Hu7, SMMC-7721, and BEL-7402, a lowly aggressive HCC cell line (MHCC97-L), or highly aggressive HCC cell lines (malignant cells) MHCC97-H was purchased from Type Culture Collection of the Chinese Academy of sciences (Shanghai, P.R. China). Cells were cultured in DMEM (Dulbecco's Modified Eagle Medium) with $10 \%$ fetal bovine serum (FBS). The cDNA samples derived from HCC clinical specimens were gifts from Dr. Fan Feng in No. 302nd hospital, Chinese PLA. The HCC clinical specimens were collected and obtained with the informed consent of patients and with approval for experiments from No. 302nd hospital, Chinese PLA. A total of $52 \mathrm{HCC}$ cases were included (Supplementary Table 1$)^{33,34}$. Antitumor agents: Betulin was purchased from MCE Corporate (NJ, USA, Cat. No. HY-N0083); Sorafenib was purchased from Selleck Corporation
(Houston, TX, USA, Cat. No. S7397). The expression vector of SREBP-1 and SREBP-1 siRNA was purchased from Vigene Corporation, Jinan, China. The siRNA sequence of SREBP-1 is 5'-GCUCCUCACUUGAAGGCUUTT-3'. A lentivirus particle of siSREBP-1 was prepared by Vigene Corporation, Jinan, China.

\section{Real-time quantitative PCR}

After treating the cells with a series of concentrations of Betulin or Sorafenib (Supplementary Table 2), the RNA samples of the cells were collected and reverse transcribed into cDNA according to the manufacturer's instructions (Thermo Fisher Scientific, Waltham, MA, USA). For cDNA samples derived from clinical specimens and cells, quantitative PCR assays were performed according to the manufacturer's instructions (Thermo Fisher Scientific, Waltham, MA, USA) and methods described by Liang et al. and Ji et al. ${ }^{35,36}$. For primers used in quantitative PCR detection, see Supplementary Table 3.

\section{Western blot experiments}

For cell-based experiments, L-02, HepG2, MHCC97-H, MHCC97-L, BEL-7402, SMMC-7721, and Hu7 cells were cultured and harvested after the indicated times. For animal experiments, tumor tissues were harvested and digested. Protein samples were extracted from cells and analyzed for SDS-PAGE. Then, protein samples were trans-printed into PVDF membranes. Next, the membranes were blocked by $5 \%$ BSA diluted with TBST at $37^{\circ} \mathrm{C}$ for $2 \mathrm{~h}$. After blocking, the membranes were in turn incubated by primary antibodies (anti-SREBP-1 antibody [Santa Cruz, USA] or anti- $\beta$-Actin antibody [Abcam Corporation, UK]) and the secondary antibody (HRPcoupling antibody, Abcam Corporation, UK). The antibodies of PARP or ki67 were described in our previous work. Membrane exposure was performed in chemiluminescence by using an ECL kit (Amersham Biosciences, Piscataway, NJ, USA $)^{37,38}$.

\section{MTT assay}

After treating HCC cells with the indicated concentrations of agents (Supplementary Table 3), the MTT assay was performed. The amount of HCC cells was determined by the absorbance values of the cell samples at a wavelength of $490 \mathrm{~nm}$. On this basis, the inhibition rate of the drug action was calculated by the OD values. The calculation formula of the inhibition rate is (absorbance value at $490 \mathrm{~nm}$ in the control group-absorbance at $490 \mathrm{~nm}$ in the experimental group)/ absorbance at the wavelength of $490 \mathrm{~nm}$ in the control group $\times 100 \%^{39-41}$.

\section{In vitro cell migration and invasion}

After the HCC cells were transfected with the expression vectors or treated with the indicated concentration of 
agents, the cells were collected for Transwell experiments: the Transwell chamber was pre-plated with ECM glue, and the cells were added to the chamber, fixed for $12-16 \mathrm{~h}$, stained, and photographed. For the obtained photographs, quantitative analysis was performed by using image analysis software Image $\mathrm{J}^{42,43}$. The specific calculation formula is relative invasion/migration cell number $=$ cell total $\mathrm{pixel} /$ image total pixel; drug inhibition rate $=$ (control group relative invasion/migration cell number-experiment group) / (control group relative invasion/migration cell number $) \times 100 \%$.

\section{Metabolic examinations}

Metabolic examinations were carried out according to the methods provided by $\mathrm{Li}$ et al. ${ }^{44,45}$. Glycolytic activity examinations were performed in either cultured tumor cells or cells isolated from tumors. The glucose uptake (Glucose Uptake Assay Kit (Colorimetric), (ab136955), Abcam), lactate (Lactate-Glo ${ }^{\mathrm{Tx}}$ Assay Kit, Promega), and ATP (ATP Assay Kit (Colorimetric/Fluorometric) (ab83355), Abcam) production, LDH activity (Lactate Dehydrogenase Activity Assay Kit, Cat\#: MAK066, Sigma), extracellular acidification rate (ECAR, Seahorse XF Glycolysis Stress Test Kit, Agilent), and oxygenconsumption rate (OCR, Seahorse XF Cell Mito Stress Test Kit, Agilent) were measured according to the manufacturer's instructions. On this basis, the inhibition rate was calculated: (control group biochemical index-experimental group biochemical index)/(control group biochemical index) $\times 100 \%$.

\section{Subcutaneous HCC tumor model}

All animal experiments and protocols were approved by the Animal Care and Use Committee of the General hospital, Chinese PLA, and all animal experiments were carried out in accordance with the UK Animals (Scientific Procedures) Act, 1986 and its associated guidelines. The 4-6-week-old nude mice (Bal B/c mice with $\mathrm{T}$ cell/thymus deletion features) were used. HCC cells were cultured, and the cells were injected subcutaneously into nude mice. Then, antitumor agents were intragastrically administered 2-3 days after injection of HCC cells ${ }^{46,47}$. The concentration gradient of the agents used in the subcutaneous tumor formation experiments is shown in Supplementary Table 4. Animals were intragastrically administered with different concentrations of Betulin, Sorafenib, or Betulin + Sorafenib. The drug was administered once per 2 days, and after 10 times' treatments (21 days), the animals were killed to collect tumor tissue. The tumor size was calculated and the tumor was weighed. The inhibition rate of the drug acting on the subcutaneous tumor formation of HCC cells was calculated according to tumor size and tumor weight: the inhibition rate calculated based on tumor size [(control tumor size)-(tumor size of the drug treatment group)]/ (control tumor size) $\times 100 \%$; the inhibition rate calculated based on tumor weight [(control tumor weight)-(tumor weight of the drug treatment group)]/(control group tumor weight) $\times 100 \%$.

\section{Intrahepatic tumor models in nude mice}

The collected HCC subcutaneous tumors were prepared into tissue microblocks, and the tissue microblocks were transplanted into the liver of the nude mice ${ }^{48,49}$. After 2-3 days of tumor transplantation, oral administration of agents was performed, and a solvent control, Betulin or Sorafenib was administered. After 4-6 weeks, the animals were imaged in vivo. Nude mice were inhaled with isoflurane, and the nude mice were injected with $200 \mu \mathrm{Ci}(7.4 \mathrm{MBq})$ of the ${ }^{18} \mathrm{~F}$-FDG radionuclide probe via the tail-vein injection, and the PET testing of animal was performed after about $40-50 \mathrm{~min}$. According to the results of PET, the animal's anatomy was collected to obtain liver specimens. The specimen was photographed, and the image analysis software Image J was used to analyze the image to determine the relative area of the tumor lesions: [(the total number of pixels in the tumor lesion)/(the total number of pixels in the image)]/[(the total number of pixels in the liver lesion)/(total number of pixels in the image)] $\times 100 \%$; using the Geiger counter to analyze the intensity of the specimen, the liver nuclide intensity is (unit weight of liver organ nuclides)/(unit weight of blood nuclides). The inhibition rate calculated based on the relative area of the tumor lesions [(relative area of tumor lesions in the control group) - (relative area of tumor lesions in the drug treatment group)]/(relative area of tumor lesions in the control group) $\times 100 \%$; based on tumor weight, the calculated inhibition rate was [(control liver nuclide intensity)-(hepatic nuclide intensity in the drug treatment group)]/(control liver nucleus intensity) $\times 100 \%^{50-52}$.

\section{Statistical analysis}

Statistical analysis was performed by Bonferroni's correction with or without two-way ANOVA by using SPSS statistical software (IBM Corporation, Armonk, NY, USA). The $\mathrm{IC}_{50}$ and $\mathrm{EC}_{50}$ value of each agent was calculated by the Origin (Origin 6.1; OriginLab Corporation, Northampton, MA, USA). A $P$-value of $<0.05$ was considered statistically significant.

\section{Results}

SREBP-1 level negatively correlates to the prognosis of HCC patients

The expression of SREBP-1 in 52 fine-needle aspiration tumor specimens derived from HCC patients who received Sorafenib treatment was measured and analyzed. Then these patients were divided into two groups 

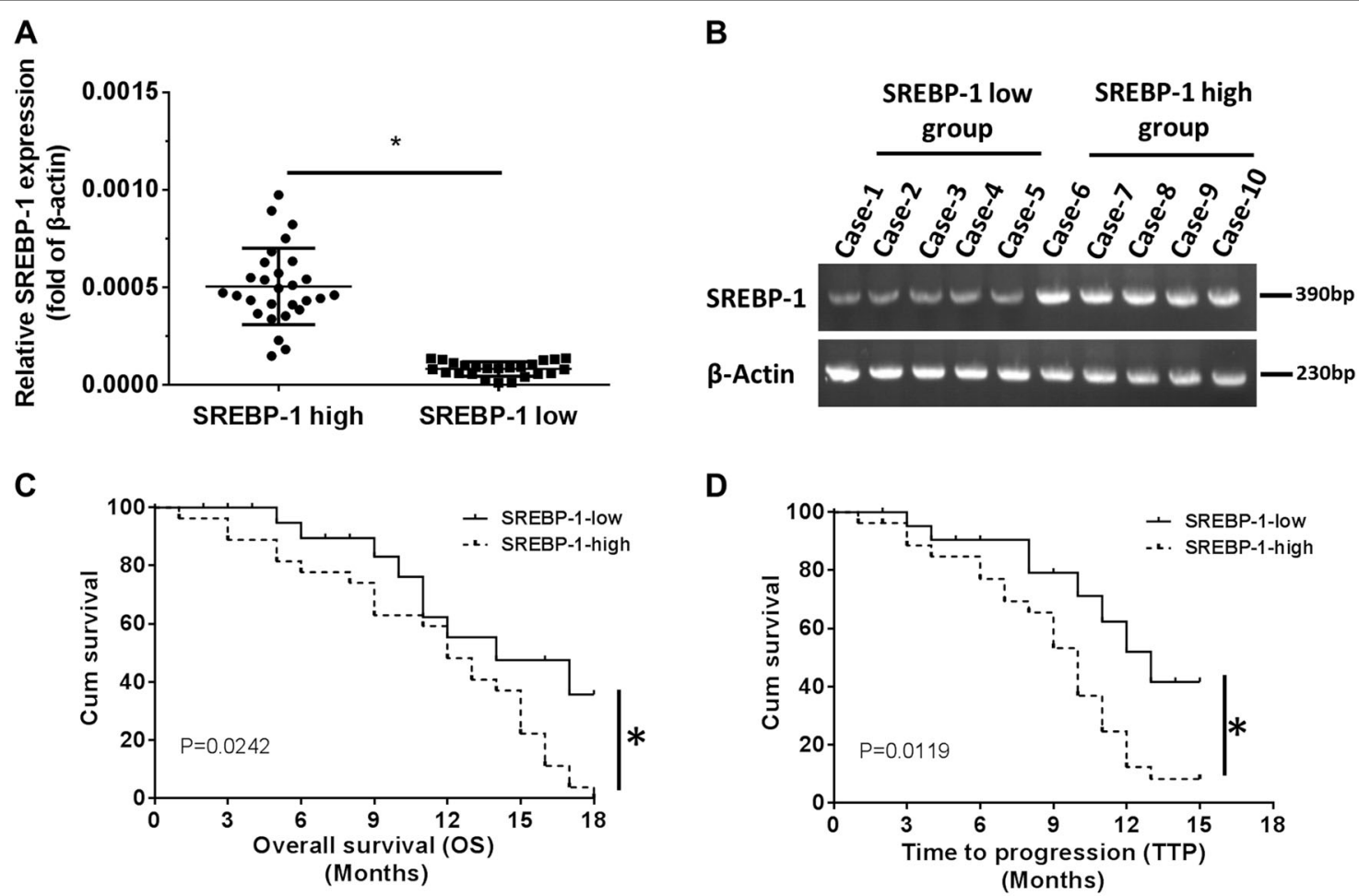

D

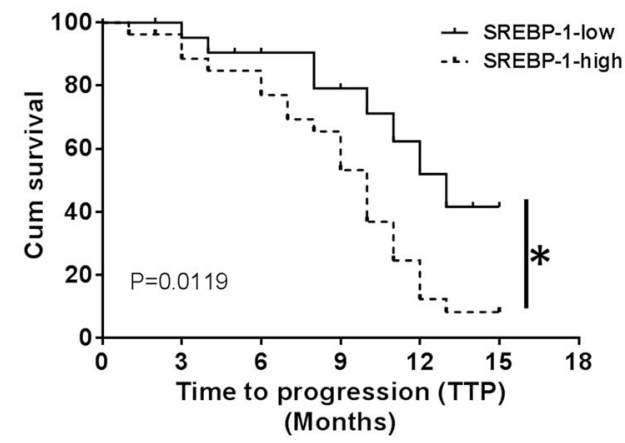

E

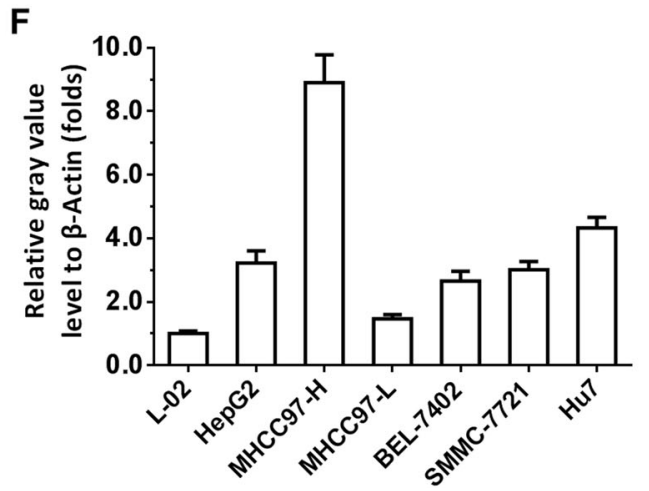

Fig. 1 High SREBP-1 level correlates to poor prognosis of advanced HCC patients who received Sorafenib treatment. a Patients were divided into SREBP-1 high and SREBP-1 low groups based on the expression level. b PCR results from ten representative specimens (five high and five low). $\mathbf{c}$ Overall survival (OS) comparison of HCC patients in the SREBP-1 high group and the SREBP-1 low group. $\mathbf{d}$ Time to progress (TTP) of HCC patients in the SREBP-1 high group and the SREBP-1 low group. $\mathbf{e}$, $\mathbf{f}$ The relative SREBP-1 expressions at both MRNA (e) and protein levels (f) in different cell lines. Survival analysis was performed by the Kaplan-Meier method and compared by the log-rank test. Paired samples were tested by the paired-sample $t$ test. Significant: ${ }^{*} p<0.05$ in all figures

according to the median value of the SREBP-1 expression: high and low SREBP-1 groups (Fig. 1a, b). After analysis, we found that the prognosis of patients with low SREBP-1 levels was better than that of patients with high SREBP-1 levels (Fig. 1c, d). The time to progression (TTP) and the overall survival (OS) time of the high SREBP-1 group were shorter compared with those of the low SREBP-1 group (Table 1), suggesting that SREBP-1 negatively correlates to HCC treatment outcomes. In addition, we found that the level of SREBP-1 in HCC cells was significantly higher than that of L-02 nonmalignant liver cells (Fig. 1e, f). High aggressive MHCC97-H cells have the highest SREBP-1 expression among the tested HCC cell lines, suggesting that SREBP-1 correlates with a higher metastatic capacity.

\section{SREBP-1 promotes HCC cell proliferation and metastasis}

We further tested the role of SREBP-1 in the proliferation and metastasis of HCC cells. Knockdown of SREBP-1 expression in MHCC97-H cells led to an inhibited proliferation and metastasis (Supplementary 
Table 1 SREBP-1 expression and outcome of sorafenib treatment

\begin{tabular}{llll}
\hline & \multicolumn{2}{c}{ SREBP-1 mRNA expression } & \multicolumn{1}{c}{$\boldsymbol{P}$} \\
\cline { 2 - 3 } & \multicolumn{1}{c}{ High $(\boldsymbol{n}=\mathbf{2 7})$} & Low $(\boldsymbol{n}=\mathbf{2 5})$ & \\
\hline TTP & 9.1 & 11.8 & 0.012 \\
& $7.7-10.4(\mathrm{M})$ & $10.1-13.5(\mathrm{M})$ & \\
OS & 11.3 & 13.7 & 0.024 \\
& $9.4-13.1(\mathrm{M})$ & $11.6-15.8(\mathrm{M})$ & \\
Overall response rate (CR + PR) & $1(3.7 \%)$ & $3(12 \%)$ & \\
Disease control rate (CR + PR + & $5(18.5 \%)$ & $8(32 \%)$ & \\
SD) & & & \\
\hline
\end{tabular}

TTP time to progress, $O S$ overall survival, $P R$ partial remission, $C R$ complete remission, $S D$ stable disease, $M$ months

Fig. 1). Accordingly, SREBP-1 overexpression in MHCC97-L cells, which has the lowest SREBP-1 expression level among the tested $\mathrm{HCC}$ cell lines, promoted cell proliferation and metastasis (Supplementary Fig. 2). Similarly, SREBP-1 inhibition through its inhibitor Betulin in MHCC97-H cells mimicked the effects of gene knockdown (Supplementary Fig. 3A-C). To further verify the specificity of Betulin, we constructed a luciferase reporter gene vector which harbored a SREBP-1-binding element, transfected MHCC97-H cells with the reporter vectors, and performed Betulin or vehicle administration. We found that Betulin treatment decreased the luciferase activity in a dose-dependent manner, compared with the vehicle control (Supplementary Fig. 3D). Taken together, these results validate that SREBP-1 promotes HCC cell proliferation and metastasis, and the SREBP-1 inhibitor Betulin blocks SREBP-1's transcription factor activity specifically.

\section{Knockdown or inhibition of SREBP-1 thwarts the glycolytic activity of HCC cells}

Next, we tested the role of SREBP-1 in the regulation of glycolytic activity of HCC cells. Knockdown of SREBP-1 by siRNA decreased glucose uptake and lactate dehydrogenase (LDH) activity in MHCC97-H cells (Supplementary Fig. 4A, B), suggesting that SREBP-1 downregulation impairs anaerobic glycolytic activity. Accordingly, reduced ATP and lactate productions were found upon SREBP-1 knockdown (Supplementary Fig. 4C, D). Moreover, in the SREBP-1-overexpressed MHCC97-L cells, we detected higher glucose uptake, increased LDH activity, and more lactate and ATP production (Supplementary Fig. 4E-H). Next, the glycolysis stress test showed that the SREBP-1 knockdown results in the decreased extracellular acidification rate (ECAR), indicating a lower overall glycolytic activity (Fig. 2a).
Similarly, SREBP-1 overexpression induced a higher ECAR in MHCC97-L cells (Fig. 2b), suggesting the regulatory role of SREBP-1 on HCC cell glycolysis. As an opposite oxidative phosphorylation activity is often observed upon alterations of glycolysis occurrence in tumor cells, which was also termed as the Warburg effect, we performed mitochondrial respiration tests for the oxygen-consumption rate (OCR) measurement. Our results showed increased OCR in SREBP-1 knockdown, whereas decreased OCR in SREBP-1 overexpression groups (Fig. 2c, d). Administration of the SREBP-1 inhibitor Betulin on MHCC97-H cells showed the similar effects, compared with the SREBP-1 knockdown, on lactate and ATP production, and glycolytic activity (Supplementary Fig. 5). Taken together, these data suggest that knockdown or inhibition of SREBP-1 dampens the glucose uptake, anaerobic glycolytic activity, and ATP production of HCC cells.

By testing the transcription level of the key genes in metabolism pathways, we found that Betulin treatment reduced the expression of lipid metabolism-associated genes, including acetyl-CoA carboxylation (ACC), ATP citrate lyase (ACLY), fatty acid synthase (FASN), and acylCoA synthetase (ACS) as expected (Fig. 2e, Supplementary Fig. 6 and Supplementary Table 5). For glucose metabolism, glucose uptake, and hypoxia stress, we found decreased expression of GLUT1, LDHA, HIF- $1 \alpha$, and EPAS-1 genes, which is in line with our discovery in the present work. Moreover, given that an abnormal Warburg effect in malignant cells may alter the EMT, which induces the resistance of HCC cells to antitumor chemotherapies, we tested EMT marker genes Twist, Snail, N-cadherin, and Vimentin, and found that they were downregulated after Betulin treatment, indicating a correlation between SREBP-1 and chemoresistance.

SREBP-1 enhances the sensitivity of HCC cells to Sorafenib

To study the role of SREBP-1 in chemoresistance, we performed SREBP-1 overexpression, knockdown, or inhibition in HCC cells in the presence of Sorafenib treatment. Silence of SREBP-1 in MHCC97-H cells enhanced the cytotoxic effect of Sorafenib; the $\mathrm{IC}_{50}$ value decreased from $0.57 \pm 0.07$ to $0.12 \pm 0.01 \mu \mathrm{mol} / \mathrm{L}$ (Fig. 3a). The resistance index (RI) was 4.97. On the other hand, overexpression of SREBP-1 in MHCC97-L cells resisted better to Sorafenib; the $\mathrm{IC}_{50}$ values increased from $0.78 \pm$ 0.04 to $3.33 \pm 0.23 \mu \mathrm{mol} / \mathrm{L}$ (Fig. 3b). Similarly, SREBP-1 knockdown synergized the effect of Sorafenib on cell metastasis (Supplementary Fig. 7). Moreover, Betulin administration augmented the killing effect of Sorafenib on MHCC97-H cells, with a decline of $\mathrm{IC}_{50}$ from $0.58 \pm$ 0.06 to $0.15 \pm 0.02 \mu \mathrm{mol} / \mathrm{L}$ (Fig. 3c), and facilitated Sorafenib-mediated suppression on metastasis (Fig. 3d). These in vitro data indicate that SREBP-1 induces 

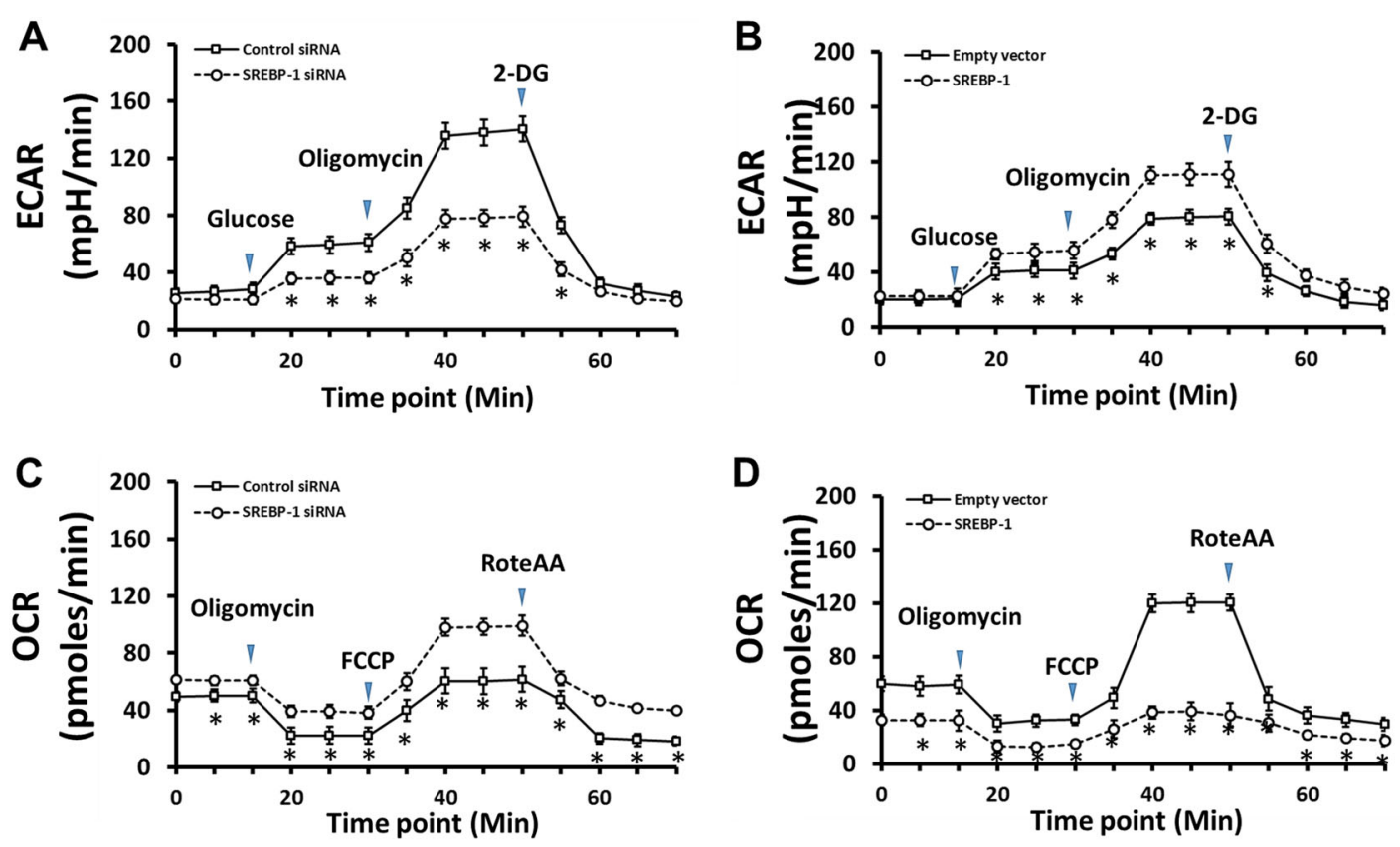

E

Betulin concentration ( $\mu \mathrm{mol} / \mathrm{L})$

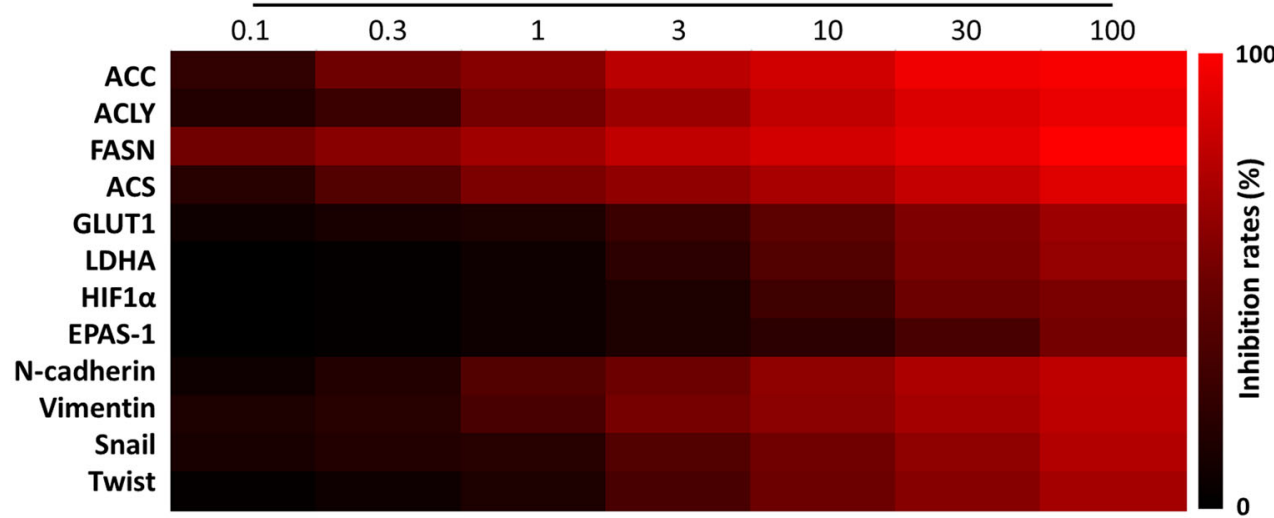

Fig. 2 SREBP-1 regulates the glycolytic activity of HCC cells. a Extracellular acidification rate (ECAR) measurement in high metastatic MHCC97-H cells transfected with control or SREBP-1 siRNAs. b ECAR measurement in low metastatic MHCC97-L cells transfected with empty or SREBP-1expressing vectors. c Oxygen-consumption rate (OCR) measurement in MHCC97-H cells from a. d OCR measurement in MHCC97-L cells from c. e MHCC97-H cells were treated with the indicated concentrations of Betulin $(100,30,10,3,1,0.3$, or $0.1 \mu \mathrm{mol} / \mathrm{L})$. Next, the cells were harvested for quantitative RT-PCR. The inhibition rates of Betulin on gene expression were calculated and shown by a heatmap

Sorafenib resistance in HCC cells, suggesting the inhibition of SREBP-1 as a strategy to overcome chemoresistance.

Betulin facilitates Sorafenib's antitumor effect on s.c. HCC tumors

We next examined the effect of SREBP-1 inhibition on Sorafenib's effects in vivo. We first found that Betulin oral gavage administration significantly suppressed s.c. MHCC97-H tumor growth (Supplementary Fig. 8). Gene expression analysis showed that Betulin treatment decreased glucose uptake, LDH activity, and lactate and ATP production in tumor cells in a dose-dependent manner (Supplementary Fig. 9A, B). Furthermore, gene expression analysis of isolated tumor cells showed that Betulin treatment reduced fatty acid and glucose metabolisms and EMT in tumors (Supplementary Fig. 9C and Supplementary Table 6). In order to test whether Betulin works synergistically with Sorafenib on HCC tumors, we administered Betulin at $2 \mathrm{mg} / \mathrm{kg}$, the mild concentration which did not have an obvious tumor rejection effect but had SREBP-1 inhibition activity, in the following experiments. 


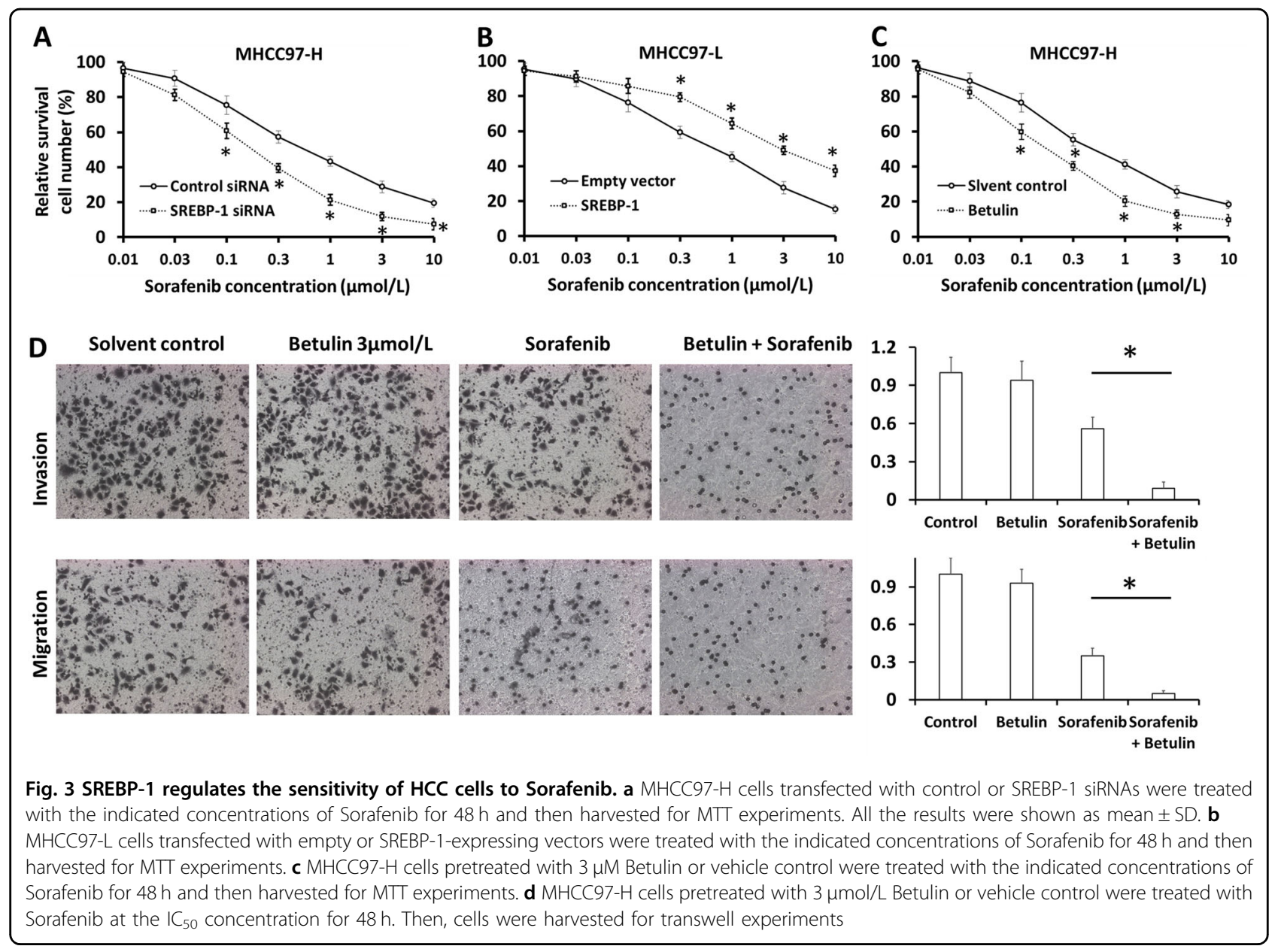

When combined with Sorafenib, Betulin significantly enhanced the growth of MHCC97-H cells in s.c. tumors, compared with Sorafenib treatment (Fig. 4). This result suggests that Betulin and Sorafenib synergistically reject HCC tumors. We next examined the specificity of Betulin on promoting Sorafenib's antitumor effect in vivo. We administered HCC s.c. tumors with SREBP-1 siRNA during Sorafenib or Sorafenib and Betulin combination treatments, and found that Betulin could not further control the growth of SREBP-1 knockdown tumors (Fig. $5 \mathrm{a}, \mathrm{b})$. However, the effect induced by SREBP-1 knockdown was rescued by ectopic expression of a mutated form of SREBP-1 (SREBP-1 ${ }^{\text {mut }}$ ), which cannot be targeted by the siRNA (Fig. 5a, b). On the other hand, SREBP-1overexpressed HCC tumors resisted to Sorafenib treatment, but were susceptible to Sorafenib and Betulin combinations (Supplementary Fig. 10A, B). These observations suggest that Betulin facilitates Sorafenib's effect through SREBP-1 inhibition specifically. Sorafenib induces tumor regression through tumor cell apoptosis. Thus, we tested the level of pro-PARP, cleaved-PARP, and Ki67 in s.c. tumors treated as described above. We found that the knockdown of SREBP-1 promoted apoptosis and restricted cell proliferation, whereas SREBP-1 overexpression reduced cell apoptosis (Fig. 5c and Supplementary Fig. 10C).

\section{Betulin improves Sorafenib-mediated blockade of HCC tumor in situ growth}

To study the effect of Betulin on in situ HCC tumor growth during Sorafenib treatment, we established in situ HCC tumors in nude mice livers by using MHCC97-H cell implantation. We then performed Sorafenib or Sorafenib plus Betulin treatment every other day 10 times in total and in the endpoint (day 21 post injection) examined tumor burden by PET/CT scanning. The analysis of the imaging results indicated that Betulin enhanced the effect of Sorafenib upon blocking HCC in situ growth (Fig. $6 \mathrm{a}-\mathrm{c})$. Meanwhile, we collected liver tissues and measured tumor lesions (Fig. 6d). Our data showed that the tumor nodule areas of Betulin- and Sorafenib-treated mice were smaller than those of Sorafenib-treated or control mice 

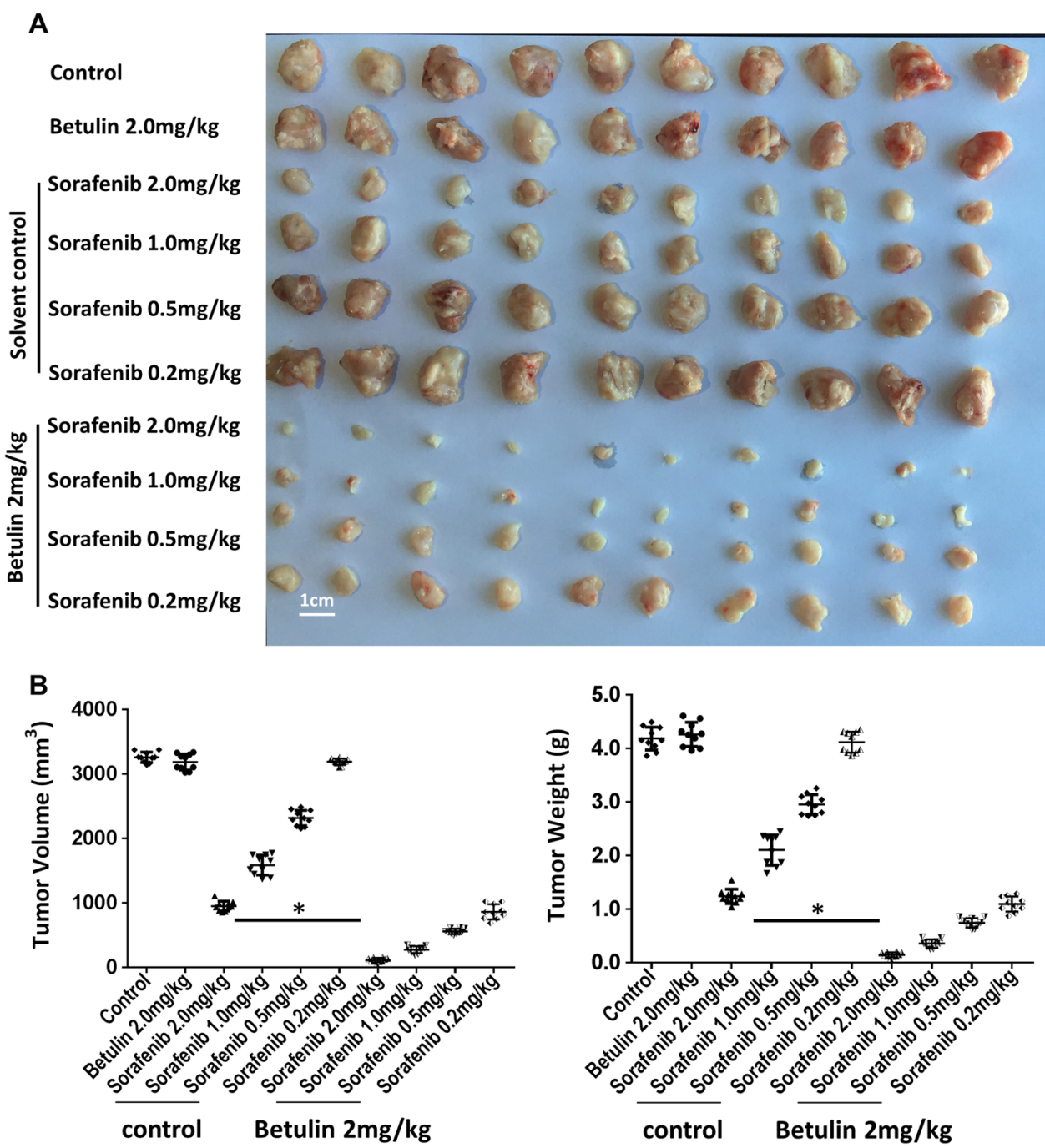

Fig. 4 Betulin synergizes Sorafenib's effect on HCC s.c. tumor growth. a MHCC97-H cells were injected into nude mice subcutaneously. At day 6, mice started receiving vehicle control, or the indicated concentrations of Sorafenib, or $2 \mathrm{mg} / \mathrm{kg}$ Betulin or the indicated concentration of Sorafenib + $2 \mathrm{mg} / \mathrm{kg}$ Betulin orally every other day 10 times. At day 21 post treatment, mice were killed and tumors were obtained $(N=10)$. b Quantitative results of tumor volume and tumor weight from a

(Fig. 6e, f). These results indicate that Betulin synergizes Sorafenib in blocking HCC in situ growth, which supports our findings in HCC s.c. tumor treatments. To further study the specificity of Betulin, we performed Sorafenib or Sorafenib plus Betulin treatment upon SREBP-1 knockdown or SREBP-1 overexpressing HCC in situ tumorbearing mice. We found that SREBP-1 knockdown diminished the effect of Betulin upon synergizing Sorafenib (Supplementary Fig. 11), whereas Betulin exerted a considerable synergistic effect upon treating SREBP-1overexpressing tumors (Supplementary Fig. 12). These results suggested that Betulin synergizes Sorafenib's effect upon controlling in situ HCC tumors by targeting SREBP-1.

\section{Discussion}

Tumors are characterized by intensive anaerobic glycolysis, which benefits tumor cells via providing quick energy for cell proliferation, altering the hostile tumor microenvironment for immune cell infiltration, and inducing drug resistance ${ }^{11,53,54}$. Therefore, targeting glucose metabolism-related pathways is considered as an effective approach to control tumor growth and enhance the efficacy of antitumor chemotherapy. SREBP-1 is one of the major regulators of cellular lipid metabolism ${ }^{25-27}$; it controls lipid synthesis via transcriptional regulation of its downstream genes: FASN, ACC, ACLY, SCD, and so on $^{25-27}$. Due to the close correlation of glucose and lipid metabolisms, SREBP-1 is also known to regulate glucose 


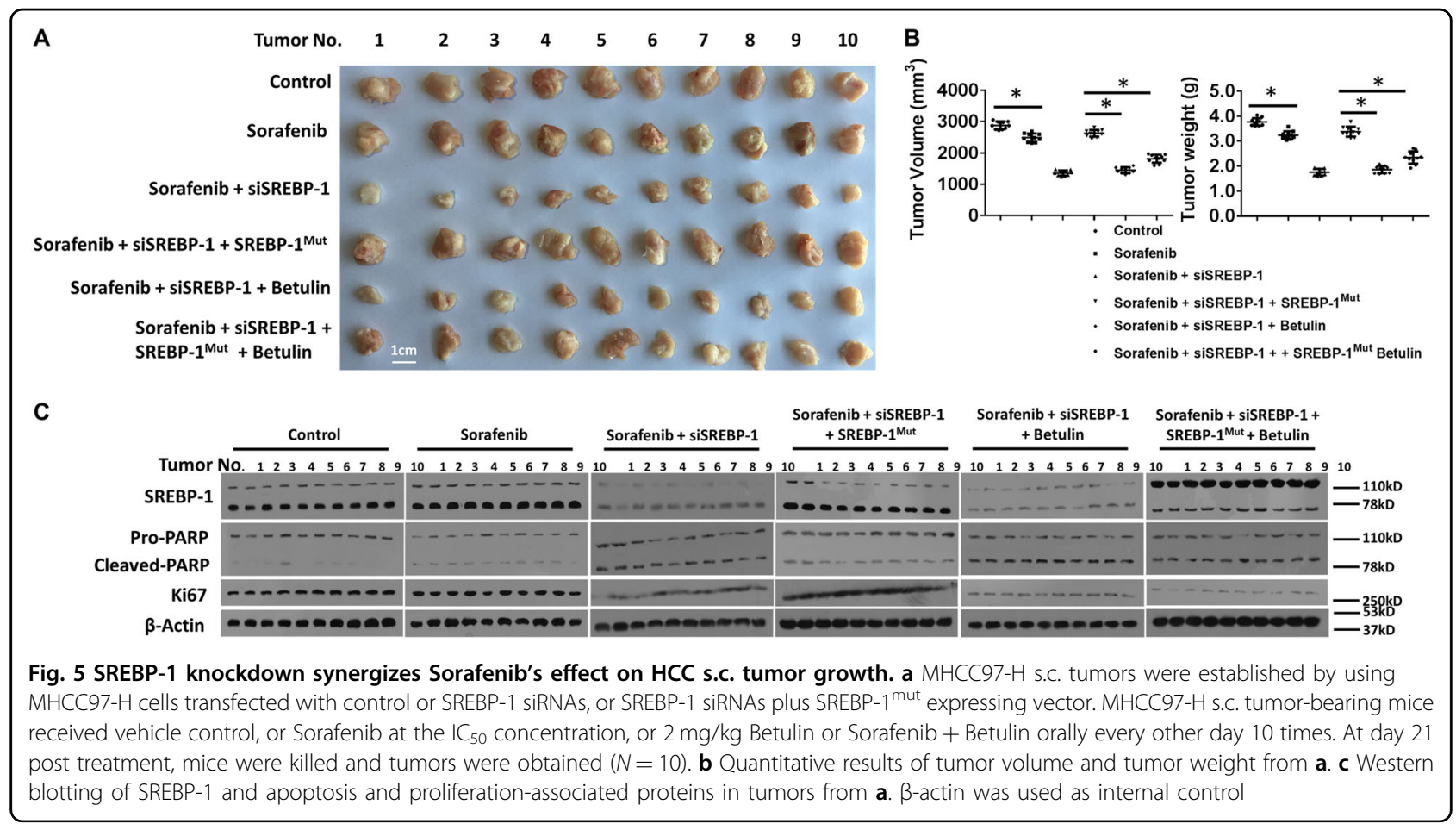

and glutamine metabolic pathways ${ }^{25-27,54}$. Betulin can inhibit SREBP family protein activation by directly binding to the SCAP region of this molecule which further blocks the cleavage of SREBP. Li et al. have reported that inhibiting SREBPs by Betulin can suppress HCC tumorassociated inflammation ${ }^{55}$. Inflammation in tumors, specifically induced by chronic viral infection, is critical for tumor-associated immune suppression and drug resistance ${ }^{56-59}$. Thus, we hypothesized that Betulin treatment on HCC tumor models can induce tumor regression and reduce drug resistance against chemotherapy. In the present study, we showed that a high level of SREBP-1 correlates with poor prognosis of HCC patients treated with the chemotherapy agent Sorafenib. Our study provides evidence that SREBP-1 protects the tumor cell via enhancing glycolytic activities and SREBP-1 inhibition induces cell death and promotes the antitumor effect of Sorafenib. These results indicated that SREBP-1 is critical for HCC tumor development and targeting to SREBP-1 is therapeutically effective for HCC treatment.

The Warburg effect is characterized by the transformation of the energy acquisition from oxidative phosphorylation to anaerobic glycolysis, which is regarded as a hallmark of tumor cells. Intracellular glucose can be hydrolyzed into pyruvate during glycolysis; then pyruvate goes into the tricarboxylic acid cycle when the oxygen supply is sufficient and energy demand is low ${ }^{11,60}$. However, in the hypoxic tumor microenvironment, where energy demand is very high, pyruvate is metabolized into lactate acid via anaerobic glycolysis, and glucose uptake is enhanced to increase the ATP production in tumor cells $^{61-63}$. To block glycolytic activity during HCC treatment, there are three strategies: (1) using inhibitors of glucose transporters ${ }^{63,64}$, (2) using inhibitors targeting hypoxia-associated pathways ${ }^{11,65-67}$, and (3) inhibitors of $\mathrm{LDHA}^{68}$ to ameliorate the accumulation of lactic acid. Hypersynthesis of fatty acid in HCC cells is driving glucose uptake ${ }^{61-65}$. Inhibition of SREBP-1 represses lipid metabolism in tumor cells, and in turn inhibits glucose uptake and glycolysis ${ }^{69-71}$. In this study, we showed that knockdown or inhibition of SREBP-1 in HCC cells impaired glucose uptake, LDH activity, and reduced ATP and lactate production, which provide mechanistic insights into the SREBP-1 function.

As a primary therapeutic choice of HCC treatment, Sorafenib does not deliver a robust effect due to the subsequent resistance that occurs soon after the initial treatment ${ }^{9}$. It has been reported that inhibition of lipid metabolism effectively represses the growth and metastasis of tumor cells and reduces Sorafenib resistance ${ }^{72-74}$. Thus, the inhibition of cellular metabolisms which facilitate tumor cell survival may enhance the efficacy of therapeutic chemodrugs. Our results indicated that Betulin as the SREBP-1 inhibitor overcomes the resistance of HCC cells to Sorafenib. Further studies will attempt to address two aspects: first, study the mechanism by which Betulin-treated cells or SREBP-1 knockdown cells become less resistant to Sorafenib; second, prove the accessibility that SREBP-1 becomes a prognosis marker of HCC patients. 


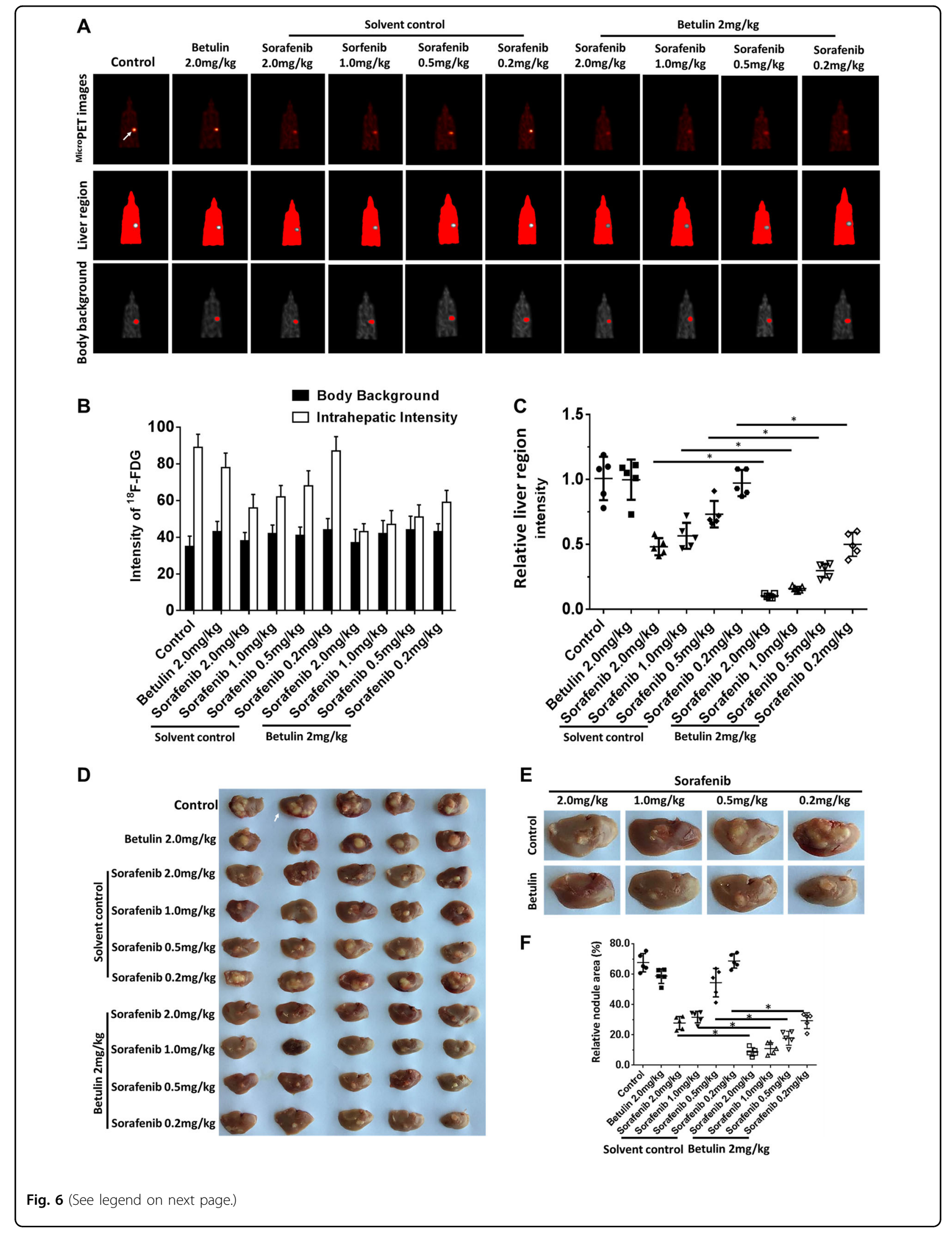


(see figure on previous page)

Fig. 6 Betulin synergizes Sorafenib's effect on HCC in situ growth in the mouse liver. a The in situ xenograft tumors were established by injected MHCC97-H cells directly into mouse livers. Tumor-bearing mice then received vehicle control, or the indicated concentrations of Sorafenib, or $2 \mathrm{mg} / \mathrm{kg}$ Betulin or the indicated concentration of Sorafenib $+2 \mathrm{mg} / \mathrm{kg}$ Betulin orally every other day 10 times. At day 21 post treatment, mice were scanned by in vivo small-animal MicroPET imaging $(N=10)$. The images show the in situ tumors. b The ${ }^{18}$ F-FDG intensity analysis and quantification results. c Relative inhibitory rate of tumor growth calculated from (b). $\mathbf{d}$ Livers from control or tumor-bearing mice from $\mathbf{a}$. $\mathbf{e}$ Representative tumor nodules from each treatment group. $\mathbf{f}$ Quantification results from $\mathbf{e}$

\section{Acknowledgements}

This work is supported by Translational Medicine Program of Chinese PLA General Hospital internal grant (2017TM-022) for F.Y.

\section{Author details}

${ }^{1}$ Department of Oncology, the Second Medical Centre \& National Clinical Research Center of Geriatric Disease, Chinese PLA General Hospital, 100853 Beijing, People's Republic of China. ${ }^{2}$ Center for Clinical Laboratory, the Fifth Medical Centre, Chinese PLA General Hospital, 100039 Beijing, People's Republic of China. ${ }^{3}$ Department of Gastroenterology, the First Medical Centre, Chinese PLA General Hospital, 100843 Beijing, People's Republic of China. ${ }^{4}$ Department of Blood Transfusion, the First Hospital of Jilin University, Changchun 130021 Jilin Province, People's Republic of China. ${ }^{5}$ Department of Immunology, H. Lee Moffitt Cancer Center \& Research Institute, 33612 Tampa, FL, USA

\section{Author contributions}

Y.C. conceived the main ideas and wrote the paper; Y.C. and F.Y. supervised the study; F.Y. F.F., and L.W. developed major methodologies, databases, reagents, and primary experiments; X.W. and Z.L. analyzed different aspects of the results.

\section{Conflict of interest}

The authors declare that they have no conflict of interest.

\section{Publisher's note}

Springer Nature remains neutral with regard to jurisdictional claims in published maps and institutional affiliations.

Supplementary Information accompanies this paper at (https://doi.org/ 10.1038/s41419-019-1884-7).

Received: 1 July 2019 Revised: 20 August 2019 Accepted: 26 August 2019 Published online: 11 September 2019

\section{References}

1. Razavi-Shearer, D. et al. Global prevalence, treatment, and prevention of hepatitis B virus infection in 2016: a modelling study. Lancet Gastroenterol. Hepatol. 3, 383-403 (2018).

2. Nayagam, S. et al. Requirements for global elimination of hepatitis B: a modelling study. Lancet Infect. Dis. 16, 1399-1408 (2016).

3. Zhang, S., Wang, F. \& Zhang, Z. Current advances in the elimination of hepatitis B in China by 2030. Front. Med. 11, 490-501 (2017).

4. Wang, F. S. et al. The global burden of liver disease: the major impact of China. Hepatology 60, 2099-2108 (2014).

5. Bray, F. et al. Global cancer statistics 2018: GLOBOCAN estimates of incidence and mortality worldwide for 36 cancers in 185 countries. CA Cancer J. Clin. 68, 394-424 (2018).

6. Forner, A., Reig, M. \& Bruix, J. Hepatocellular carcinoma. Lancet 391, 1301-1314 (2018).

7. Kudo, M. et al. Lenvatinib versus sorafenib in first-line treatment of patients with unresectable hepatocellular carcinoma: a randomised phase 3 noninferiority trial. Lancet 391, 1163-1173 (2018).

8. Bruix, J. et al. Regorafenib for patients with hepatocellular carcinoma who progressed on sorafenib treatment (RESORCE): a randomised, double-blind, placebo-controlled, phase 3 trial. Lancet 389, 56-66 (2017).
9. Roskoski., R. Jr. Properties of FDA-approved small molecule protein kinase inhibitors. Pharmacol. Res. 144, 19-50 (2019).

10. Lacal, P. M. \& Graziani, G. Therapeutic implication of vascular endothelial growth factor receptor-1 (VEGFR-1) targeting in cancer cells and tumor microenvironment by competitive and non-competitive inhibitors. Pharmacol. Res. 136, 97-107 (2018).

11. Roskoski, R. Jr. Targeting oncogenic Raf protein-serine/threonine kinases in human cancers. Pharmacol. Res. 135, 239-258 (2018).

12. Llovet, J. M. Sorafenib in advanced hepatocellular carcinoma. N. Engl. J. Med. 359, 378-390 (2008).

13. Cheng, A. L. Efficacy and safety of sorafenib in patients in the Asia-Pacific region with advanced hepatocellular carcinoma: a phase III randomised, double-blind, placebo-controlled trial. Lancet Oncol. 10, 25-34 (2009).

14. Roskoski, R. Jr. The role of small molecule Kit protein-tyrosine kinase inhibitors in the treatment of neoplastic disorders. Pharmacol. Res. 133, 35-52 (2018).

15. Roskoski, R. Jr. The role of small molecule platelet-derived growth factor receptor (PDGFR) inhibitors in the treatment of neoplastic disorders. Pharmacol. Res. 129, 65-83 (2018).

16. Zhu, Y. J. et al. New knowledge of the mechanisms of sorafenib resistance in liver cancer. Acta Pharmacol. Sin. 38, 614-622 (2017).

17. Feng, F. et al. Which is the best combination of TACE and Sorafenib for advanced hepatocellular carcinoma treatment? A systematic review and network meta-analysis. Pharmacol. Res. 135, 89-101 (2018).

18. Shen, $Y$. et al. ARHGAP4 mediates the Warburg effect in pancreatic cancer through the mTOR and HIF-1a signaling pathways. Onco. Targets Ther. 12, 5003-5012 (2019).

19. Huang, X. et al. The HGF-MET axis coordinates liver cancer metabolism and autophagy for chemotherapeutic resistance. Autophagy 15, 1258-1279 (2019).

20. Zhang, Q. et al. Thymidine phosphorylase promotes malignant progression in hepatocellular carcinoma through pentose Warburg effect. Cell Death Dis. 10, 43-59 (2019).

21. Chen, Z. et al. MiR-3662 suppresses hepatocellular carcinoma growth through inhibition of HIF-1a-mediated Warburg effect. Cell Death Dis. 9, 549-562 (2018).

22. Zhang, L. F. et al. Suppression of miR-199a maturation by HuR is crucial for hypoxia-induced glycolytic switch in hepatocellular carcinoma. EMBO J. $\mathbf{3 4}$, 2671-2685 (2015).

23. Luo, F. et al. Hexokinase II promotes the Warburg effect by phosphorylating alpha subunit of pyruvate dehydrogenase. Chin. J. Cancer Res. 31, 521-532 (2019).

24. Berthier, A. et al. Combinatorial regulation of hepatic cytoplasmic signaling and nuclear transcriptional events by the OGT/REV-ERBa complex. Proc. Natl Acad. Sci. USA 115, E11033-E11042 (2018).

25. Yang, $X$. et al. GP73 regulates Hepatic Steatosis by enhancing SCAP-SREBPs interaction. Sci. Rep. 7, 14932 (2017).

26. $\mathrm{Wu}, \mathrm{H}$. et al. MicroRNA-21 is a potential link between non-alcoholic fatty liver disease and hepatocellular carcinoma via modulation of the HBP1-p53Srebp1c pathway. Gut 65, 1850-1860 (2016).

27. Liu, L. et al. Arginine methylation of SREBP1a via PRMT5 promotes de novolipogenesis and tumor growth. Cancer Res. 76, 1260-1272 (2016).

28. Park, S. et al. Expedient synthesis of alphitolic acid and its naturally occurring $2-$ o-ester derivatives. J. Nat. Prod. 82, 895-902 (2019).

29. Xu, G. M. et al. Betulin inhibits lipopolysaccharide/d-galactosamine-induced acute liver injury in mice through activating PPAR- $\gamma$. Biomed. Pharmacother. 106, 941-945 (2018).

30. Kanhar, S. \& Sahoo, A. K. \& Mahapatra, A. K. The ameliorative effect of homalium nepalense on carbon tetrachloride-induced hepatocellular injury in rats. Biomed. Pharmacother. 103, 903-914 (2018). 
31. Tang, J. J. et al. Inhibition of SREBP by a small molecule, betulin, improves hyperlipidemia and insulin resistance and reduces atherosclerotic plaques. Cell Metab. 13, 44-56 (2011).

32. Shimano, $\mathrm{H}$. et al. Overproduction of cholesterol and fatty acids causes massive liver enlargement in transgenic mice expressing truncated SREBP-1a. J. Clin. Invest. 98, 1575-1584 (1996).

33. Shao, Z. et al. ETS-1 induces sorafenib-resistance in hepatocellular carcinoma cells via regulating transcription factor activity of PXR. Pharmacol. Res. 135, 188-200 (2018)

34. Feng, F. et al. Pregnane $X$ receptor mediates sorafenib resistance in advanced hepatocellular carcinoma. Biochim. Biophys. Acta Gen. Subj. 1862, 1017-1030 (2018).

35. Ji, Q. et al. miR-216a inhibits osteosarcoma cell proliferation, invasion and metastasis by targeting CDK14. Cell Death Dis. 8, e3103 (2017).

36. Liang, Y. et al. The EGFR/miR-338-3p/EYA2 axis controls breast tumor growth and lung metastasis. Cell Death Dis. 8, e2928 (2017).

37. Wang, L. et al. Aminophenols increase proliferation of thyroid tumor cells by inducing the transcription factor activity of estrogen receptor a. Biomed. Pharmacother. 109, 621-628 (2019).

38. Fan, Z. et al. PTK2 promotes cancer stem cell traits in hepatocellular carcinoma by activating Wnt/ $\beta$-catenin signaling. Cancer Lett. 450 132-143 (2019).

39. Li, J. et al. MicroRNA-140-3p enhances the sensitivity of hepatocellular carcinoma cells to sorafenib by targeting pregnenolone $X$ receptor. Onco. Targets Ther. 11, 5885-5894 (2018).

40. Li, F. et al. Procaspase-3-activating compound 1 stabilizes hypoxia-inducible factor $1 a$ and induces DNA damage by sequestering ferrous iron. Cell Death Dis. 9, 1025 (2018).

41. Guan, F. et al. WX-132-18B, a novel microtubule inhibitor, exhibits promising anti-tumor effects. Oncotarget 8, 71782-71796 (2017).

42. Ingersoll, M. A. et al. p66Shc regulates migration of castration-resistant prostate cancer cells. Cell. Signal. 46, 1-14 (2018)

43. Zheng, B. et al. AKT2 contributes to increase ovarian cancer cell migration and invasion through the AKT2-PKM2-STAT3/NF-KB axis. Cell. Signal. 45, 122-131 (2018).

44. Li, L. et al. miR-30a-5p suppresses breast tumor growth and metastasis through inhibition of LDHA-mediated Warburg effect. Cancer Lett. 400, 89-98 (2017).

45. Li, L. et al. Transcriptional regulation of the warburg effect in cancer by SIX1. Cancer Cell. 33, 368-385.e7 (2018).

46. $\mathrm{Xu}, \mathrm{X}$. et al. A signature motif in LIM proteins mediates binding to checkpoint proteins and increases tumour radiosensitivity. Nat. Commun. 8, 14059-14072 (2017).

47. An, L. et al. Terfenadine combined with epirubicin impedes the chemo-resistant human non-small cell lung cancer both in vitro and in vivo through EMT and Notch reversal. Pharmacol. Res. 124, 105-115 (2017).

48. Zhang, Y. et al. Novel ADAM-17 inhibitor ZLDI-8 enhances the in vitro and in vivo chemotherapeutic effects of Sorafenib on hepatocellular carcinoma cells. Cell Death Dis. 9, 743-755 (2018)

49. Chen, $Y$. et al. LINE-1 ORF-1p enhances the transcription factor activity of pregnenolone $X$ receptor and promotes sorafenib resistance in hepatocellular carcinoma cells. Cancer Manag. Res. 10, 4421-4438 (2018).

50. Meng, D. et al. MicroRNA-645 targets urokinase plasminogen activator and decreases the invasive growth of MDA-MB-231 triple-negative breast cancer cells. Onco. Targets Ther. 11, 7733-7743 (2018).

51. Xie, $H$. et al. A new apatinib microcrystal formulation enhances the effect of radiofrequency ablation treatment on hepatocellular carcinoma. Onco. Targets Ther. 11, 3257-3265, https://doi.org/10.2147/OTT.S165000. (2018). eCollection 2018
52. Wei, L. 1 et al. Novel urokinase-plasminogen activator inhibitor SPINK13 inhibits growth and metastasis of hepatocellular carcinoma in vivo. Pharm. Res. 143, 73-85 (2019).

53. Serviddio, G., Bellanti, F. \& Vendemiale, G. Free radical biology for medicine: learning from nonalcoholic fatty liver disease. Free Radic. Biol. Med. 65, 952-968 (2013).

54. Inoue, J. \& Sato, R. New insights into the activation of sterol regulatory element-binding proteins by proteolytic processing. Biomol. Concepts $\mathbf{4}$, 417-423 (2013).

55. Li, N. et al. Inhibition of the sterol regulatory element-binding protein pathway suppresses hepatocellular carcinoma by repressing inflammation in mice. Hepatology 65, 1936-1947 (2017).

56. Mahmoudian-Sani, M. R. et al. MicroRNA-122 in patients with hepatitis B and hepatitis B virus-associated hepatocellular carcinoma. J. Gastrointest. Oncol. 10, 789-796 (2019).

57. Zhao, B. et al. MiRNA-124 inhibits the proliferation, migration and invasion of cancer cell in hepatocellular carcinoma by downregulating IncRNA-UCA1. Onco. Targets Ther. 12, 4509-4516 (2019).

58. Liang, D. Y. et al. Hepatitis B X protein upregulates decoy receptor 3 expression via the PI3K/NF-KB pathway. Cell. Signal. 62, 109346 (2019).

59. Kim, T. S. et al. Hepatitis B virus DNA levels and overall survival in hepatitis Brelated hepatocellular carcinoma patients with low-level viremia. J. Gastroenterol. Hepatol. https://doi.org/10.1111/jgh.14750 (2019).

60. Zhang, S. et al. Metabolic reprogramming links chronic intestinal inflammation and the oncogenic transformation in colorectal tumorigenesis. Cancer Lett. 450, 123-131 (2019).

61. Xu, X. D. et al. Warburg effect or reverse warburg effect? a review of cancer metabolism. Oncol. Res. Treat. 38, 117-122 (2015).

62. da Veiga Moreira, J. et al. Metabolic therapies inhibit tumor growth in vivo and in silico. Sci. Rep. 9, 3153-3162 (2019).

63. Siebeneicher, $\mathrm{H}$. et al. Identification and optimization of the first highly selective GLUT1 inhibitor BAY-876. Chem. Med. Chem. 11, 2261-2271 (2016).

64. Hanahan, D. \& Weinberg, R. A. Hallmarks of cancer: the next generation. Cell 144, 646-674 (2011)

65. Dong, $Z$ et al. Transcriptional activation of SIRT6 via FKHRL1/FOXO3a inhibits the Warburg effect in glioblastoma cells. Cell. Signal. 60, 100-113 (2019).

66. Yang, W. et al. Knockdown of CZNF292 suppressed hypoxic human hepatoma SMMC7721 cell proliferation, vasculogenic mimicry, and radioresistance. Cell. Signal. 60, 122-135 (2019).

67. Hu, Z. et al. FOXO3a-dependent up-regulation of Mxi1-0 promotes hypoxiainduced apoptosis in endothelial cells. Cell. Signal. 51, 233-242 (2018).

68. Li, L. et al. Identification and functional characterization of Lys-trimethylation of lactate dehydrogenase A. OncoTargets Ther. 12, 5395-5404 (2019).

69. Cohen, J. C., Horton, J. D. \& Hobbs, H. H. Human fatty liver disease: old questions and new insights. Science 332, 1519-1523 (2011).

70. Tennant, D. A., Duran, R. V. \& Gottlieb, E. Targeting metabolic transformation for cancer therapy. Nat. Rev. Cancer 10, 267-277 (2010).

71. Li, C. et al. SREBP-1 has a prognostic role and contributes to invasion and metastasis in human hepatocellular carcinoma. Int. J. Mol. Sci. 15, 7124-7138 (2014).

72. Sounni, N. E. et al. Blocking lipid synthesis overcomes tumor regrowth and metastasis after antiangiogenic therapy withdrawal. Cell Metab. 20, 280-294 (2014).

73. Siqingaowa et al. Sterol regulatory element-binding protein 1 inhibitors decrease pancreatic cancer cell viability and proliferation. Biochem. Biophys. Res. Commun. 488, 136-140 (2017).

74. Meyer, T. et al. Sorafenib in combination with transarterial chemoembolisation in patients with unresectable hepatocellular carcinoma (TACE 2): a randomised placebo-controlled, double-blind, phase 3 trial. Lancet Gastroenterol. Hepatol. 2, 565-575 (2017) 